\title{
Spatial Distribution Shift of Creative Industries During COVID-19 Pandemic
}

\author{
Rostislav Netek $^{\mathrm{a}, *, J^{2} \text { akub Konicek }}{ }^{\mathrm{b}}$ \\ ${ }^{a}$ Dept. of Geoinformatics, Palacký University Olomouc, Czech Rep., rostislav.netek@upol.cz \\ ${ }^{b}$ Dept. of Geoinformatics, Palacký UniversityOlomouc, Czech Rep., jakub.konicek@upol.cz \\ * Corresponding author
}

Keywords: Thematic map, COVID-19, Creative Industries

\begin{abstract}
:
The long-term research on the motivations of the actors of the creative industries within the Olomouc agglomeration, Czech Rep., has been significantly influenced by the COVID-19 pandemic. In the Czech Republic, the disease caused by the coronavirus SARS-CoV-2 struck at the end of February 2020. The government gradually began to take measures against the spread of the epidemic: on 12 March, a state of national emergency was declared, and later all public establishments, including restaurants, shops, public institutions etc., were closed because of lockdown. The restrictions thus affected not only the population but also cultural and memory institutions, business entities and self-employed persons - among them, of course, also the examined entities and actors of the cultural and creative industries.

The first wave of the COVID-19 pandemic was monitored by experts from Palacký University Olomouc with the goal to analyse both spatial and non-spatial effects of the emergency mode and related multi-level quarantine measures. The list of recommendations on a local level was developed based on the detected spatial distribution of affected subjects. Spatial distribution was affected by several aspects, especially contemporary economic, social and political restrictions which influenced the market.

Since the course of the COVID-19 pandemic had a different impact across different segments, we designed the list of recommendations focus on each segment of the culture industry: IT development; architecture \& design; books \& printing; museums \& galleries; arts \& photography; audiovisual services; music \& performing. The main focus lay on map visualisation of specific segments, integrating all analysed aspects into a single map product. The single marker method was used for visualisation because it allows both simple and comprehensive identification of monitored subjects within the study area. It has been publishing in both analogue and digital form. Moreover, the map could be extended by a list of non-spatial attributes describing the positive and negative impact of crisis for each segment separately. Common denominators can be observed across the segments as well, which supplements a summary of generally valid conclusions.
\end{abstract}

Observed results could lead to improvement of the situation within the future waves of the pandemic. Actually, it was verified during the second wave of the pandemic. 